\title{
Blazhko Cycles of $\omega$ Centauri RRab Stars
}

\author{
Johanna Jurcsik \\ Konkoly Observatory of the Hungarian Academy of Sciences
}

\begin{abstract}
The present observational strategy of globular cluster variables does not favour long observational runs, which are needed to study the modulation properties of Blazhko type RR Lyrae stars. The only globular cluster for which we have enough data (both photographic and CCD) to determine modulation cycles of RRab stars is $\omega$ Centauri. Any connection between the modulation periods and other stellar or cluster parameters may serve as a new clue to the explanation of the Blazhko phenomenon.
\end{abstract}

\section{Introduction}

RR Lyrae stars are amongst the most studied and best known pulsating variables thanks to the systematic photographic surveys of globular cluster variables prior to the quick spread of CCD technique in the 80s. Although, we have a clear picture of their global evolutionary and pulsational status, there are still unsolved problems in the details. There is also no fully consistent description of the Blazhko phenomenon. The recent finding of nonradial modes in RR Lyrae stars, both from the observational (Olech et al. 1999; Alcoock et al. 2000) and theoretical (Dziembowski \& Cassisi 1999) points of view, does not help us indeed to get closer to an understanding of the Blazhko behaviour.

To study the long term (order of magnitude of 10-100 days) phase and/or amplitude modulation of these stars, extended photometric data are required which are available only in a few cases. Among field stars there are altogether 5 variables with known frequency pattern of the modulation: AH Cam (Smith et al. 1994); RV UMa (Kovács 1995); RS Boo (Nagy 1998); AR Her (Smith et al. 1999); RR Lyr (Szeidl \& Kolláth 2000). All these cases indicate that analytically the Blazhko phenomenon can be described with the appearance in the Fourier spectrum of equidistant side-peak frequencies at $\Delta f=f_{m}=1 / P_{B l}$ distant from the primary frequency and its harmonics in both directions, but not necessarily with the same height.

Regarding cluster variables, only the existence of the modulation of different percentages of variables in different clusters has been clearly shown up to now. As variables in a globular cluster represent a much more homogeneous group (both in terms of their chemical compositions and their ages) than the field stars, then if any connection between the Blazhko properties and other physical parameters exists, it will be the easiest to detect it in individual clusters.

The present observational strategy of globular cluster photometry however, does not favour to follow any type of multi-mode behaviour. The only globular 
cluster with extended enough CCD photometry to study Blazhko variables as yet is $\omega$ Cen (Kaluzny et al. 1997). These data together with earlier photographic measurements have enabled us to determine the modulation cycles of the $\omega$ Centauri Blazhko type RRab stars.

\section{Data and Method}

- All the available long term photometries (compiled in Jurcsik et al. 2001) of $\omega$ Centauri fundamental mode Blazhko variables (13 RRab stars).

- Fourier analysis (MUFRAN package: Kolláth 1990).

A least square minimization of the residuals ( $\Theta$ transforms) using different number of harmonics (4-8) of the dominant mode frequency, and 2-11 additional frequencies from a 'Blazhko type' frequency pattern.

- $\Delta f$, which appears uniformly in the $\Theta$ transforms of the different datasets of a given variable, is accepted as the true modulation frequency.

\section{Results}

Blazhko cycle lengths are determined for all the 13 stars and are compared with other known parameters of the stars. There are hints that $P_{B l}$ depends on $\langle V\rangle$ and/or $P$, or through the metallicity dependence of these parameters, possibly on $[\mathrm{Fe} / \mathrm{H}]$.

Acknowledgments. This work has been supported by the Hungarian OTKA grants No 30954 and No 24022.

\section{References}

Alcoock, C. et al. 2000, ApJ, 542, 257

Dziembowski, W. \& Cassisi, S. 1999, Acta Astronomica Vol. 49, 371

Jurcsik, J., Clement, C., Geyer, H., \& Domsa, I. 2001, AJ, 121, 951

Kaluzny, J., Kubiak, M., Szymański, A., Udalski, W., Krzemiński, W., \& Mateo, M. 1997, A\&AS, 125, 343

Kolláth, Z. 1990, The program package MUFRAN, Occ. Techn. Notes of the Konkoly Obs., No.1, http://www.konkoly.hu/staff/kollath/mufran.html

Kovács, G. 1995, A\&A, 295, 693

Nagy, A. 1998, A\&A, 339, 440

Olech, A., Kaluzny, J., Thompson, I.B., Pych, W., Krzeminski, W., \& Schwarzenberg-Czerny A. 1999, AJ, 118, 442

Smith, H., Matthews, J., Lee, K., Williams, J., Silbermann, A. \& Bolte, M. 1994, AJ, 107, 670

Smith, H., Barnett, M., Silbermann, A., \& Gay, P. 1999, AJ, 118, 572

Szeidl, B., \& Kolláth, Z. 2000, ASPCS Vol. 203, 281 